\title{
Traumatic Spinal Subdural Hematoma with Intracranial Subdural Hematoma
}

\author{
Hyun Gon Kim, MD, Tae Wan Kim, MD, Kwan Ho Park, MD, and Moon Pyo Chi, MD \\ Department of Neurosurgery, VHS Medical Center, Seoul, Korea
}

\begin{abstract}
Traumatic spinal subdural hematoma associated with intracranial subdural hematoma is a rare condition. Herein, we report the case of a 62-year-old man with lower back pain, radiating pain, and numbness in both lower extremities, without motor weakness, for 2 weeks. Lumbar magnetic resonance imaging (MRI) revealed high signal intensity on T1-weighted image (WI), and low signal intensity on T2-WI from L2 to L5. Two weeks after conservative management, follow-up lumbar MRI did not show the hematoma and his symptoms were relieved and there was no neurological deficit; therefore, he was discharged. However, subsequently, intracranial subdural hematoma increased and upper extremity motor weakness appeared. This was treated surgically. If there is no neurological deficit, conservative treatment may be a good option. Follow-up evaluation for asymptomatic cranial subdural hematoma is necessary.

(Korean J Neurotrauma 2014;10(2):146-148)
\end{abstract}

KEY WORDS: Hematoma subdural intracranial $\cdot$ Hematoma subdural spinal $\cdot$ Spinal cord injuries $\cdot$ Injuries.

\section{Introduction}

Traumatic spinal subdural hematoma (SSDH) associated with intracranial subdural hematoma is a rare condition and usually associated with head injury. Most SSDHs are caused by hematologic disease, anticoagulation therapy, lumbar puncture and epidural anesthesia. SSDH can compress the spinal cord or rootlets, which may result in motor weakness, neurogenic bladder, and neurogenic bowel. Therefore, early diagnosis and proper treatment are necessary. We report a traumatic SSDH coexisting with intracranial subdural hematoma.

\section{Case Report}

A 62-year-old man was admitted complained of lower back pain, radiating pain, and hypoesthesia in lower extremities, for 2 weeks. One month ago, he had slipped and been collided with head and back. On admission, he was alert with-

Received: August 28, 2014 / Revised: October 10, 2014

Accepted: October 12, 2014

Address for correspondence: Tae Wan Kim, MD

Department of Neurosurgery, VHS Medical Center, 53 Jinhwang-

do-ro 61-gil, Gangdong-gu, Seoul 134-791, Korea

Tel: +82-2-2225-1363, Fax: +82-2-2225-4152

E-mail: euro3399@naver.com out neurological deficit. He had no history of antiplatelet and anticoagulant medication. The serum coagulation tests were all within the normal range. Brain computed tomography scan revealed isodense subdural hematoma in right hemisphere without midline shifting (Figure 1). There is no evidence of vertebral fractures. Spine magnetic resonance imaging (MRI) revealed high signal intensity on T1-weighted image (WI) and low signal intensity on T2-WI in the L2-5 (Figure 2). Because of no neurological deficit and no prominent mass effect, the patient was treated conservatively. Two weeks after admission, SSDH in the L2-5 was disappeared in follow-up lumbar MRI (Figure 3) and his symptoms were relieved.

\section{Discussion}

SSDHs are uncommon condition and usually related with anticoagulation, lumbar puncture, and blood dyscrasia. Traumatic SSDH is rarer than spontaneous SSDH. Some traumatic SSDHs are associated with intracranial subdural hematoma. Sometimes, SSDH is also related to brain surgery. Lower cerebrospinal fluid volume during and after surgery may predispose the tearing of the bridging veins. ${ }^{9)}$

The symptom of SSDH is variable from mild back pain and radicular pain to paraplegia. Hematomas are most likely ve- 


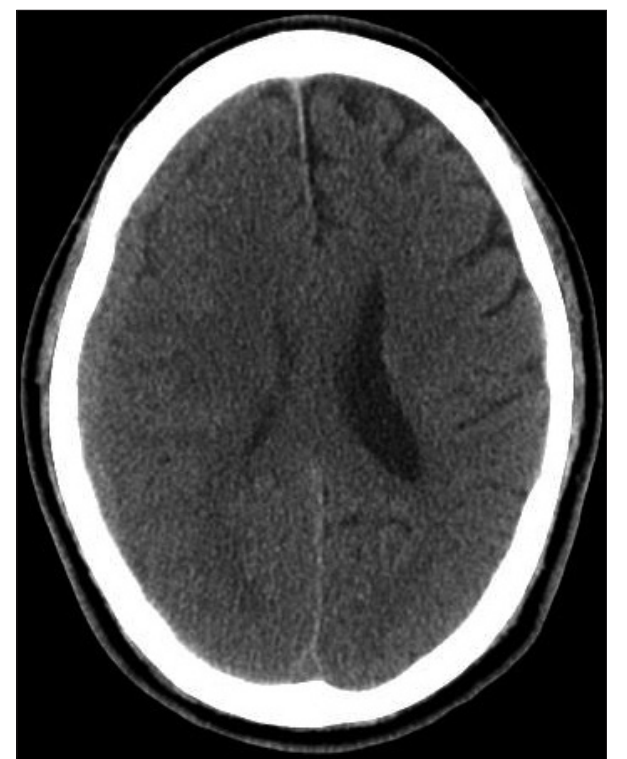

FIGURE 1. On admission, initial brain computed tomography scan showing isodense subdural hematoma.
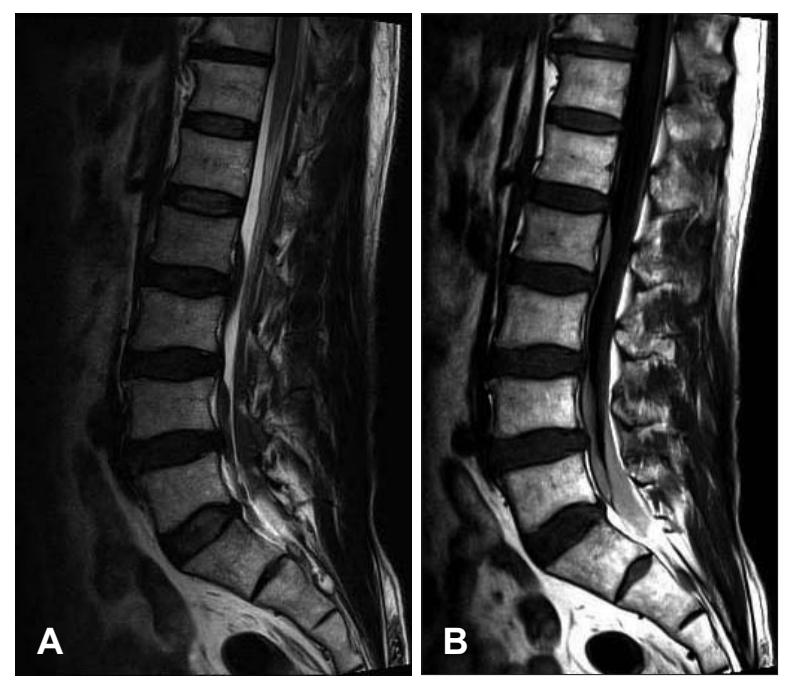

FIGURE 2. Lumbar magnetic resonance images show focal subdural fluid collection from L2 to L5 level with low signal intensity in T2-weighted image (A) and high signal intensity in T1-weighted image (B).

nous in origin, which explains the relatively slow onset of symptom. ${ }^{10)}$ If a patient with cranial subdural hematoma complains of back pain, radicular pain and weakness of extremities, then, spinal evaluation should be performed.

The mechanism of traumatic SSDH is unclear. There are no bridging veins in spinal subdural space. Although external dura is strong, the inner dura is weak and vulnerable to injury. SSDH may be related to redistribution of blood from the supratentorial subdural space to the most dependent areas due to gravity. ${ }^{1,6,8,9,16)}$ Blood moved caudally through the spinal subdural component is accumulated in the most de-

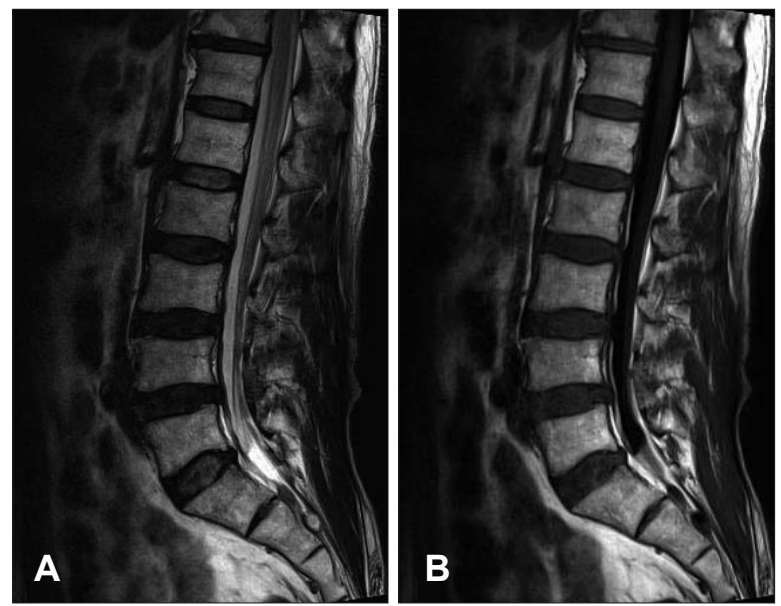

FIGURE 3. Two weeks later, follow-up sagittal T2 (A) and T1 (B) images show markedly decreased volume and mass effect of hematoma.

pendent portion of the spine. In our case, intracranial subdural hematoma increased after the absorption of SSDH. SSDH appeared a few days after the spontaneous resolution of intracranial hematoma. ${ }^{1)}$ Another theory is that the pressure from cranial space may increase shearing force between spinal subdural and subarachnoid spaces, so the inner dura may tear and bleed. ${ }^{5)}$ However, most of the intracranial hematomas are not concomitant with SSDH and the occurrence of SSDH is not necessarily proportional to the amount of intracranial hematoma. Therefore, other mechanisms that block the redistribution of subdural hematoma may exist.

Prompt diagnosis is important because early diagnosis with progressive neurological deficit before any irreversible changes is associated with a better outcome. ${ }^{5,13,15)} \mathrm{MRI}$ is valuable for diagnosing the presence, location, degree of compression by hematoma, and extent of $\mathrm{SSDH},{ }^{2)}$ and for follow-up.

The optimal treatment of SSDH is uncertain. Laminectomy and evacuation of hematoma may be one of the treatment methods. ${ }^{1,4,14)}$ Lumbar drainage is also an effective treatment method. ${ }^{3)}$ Surgery for SSDH should be indicated only for patients with moderate or severe paraparesis and sphincter disturbance. ${ }^{11,17)}$ Patients with paraplegia and sphincter disturbance have poor prognosis regardless of surgical or conservative management. $^{12)}$

Conservative treatment plays a role in the treatment of SSDH with mild symptoms. ${ }^{5,12}$ In cases where the hematoma is extensive, conservative treatment may be an option. There is no spinal cord in lumbosacral region, so the patient with lumbosacral subdural hematoma and no neurological deficit can be readily treated conservatively. ${ }^{5)}$ Our patient was also treated conservatively and had a good prognosis.

Complete resolution of SSDH was confirmed by MRI ob- 
tained 2-5 months later. In our case, SSDH was absorbed within a month from the trauma, which was more rapid than intracranial subdural hematoma. Relatively small amount of blood may be influenced rapid resolution of hematoma than other cases or there may be an individual difference of velocity of hematoma dilution by cerebrospinal fluid. Intracranial hematoma may grow larger after the absorption of SSDH because of the presence of bridging veins, normally presented cerebrospinal fluid in the intracranial subdural space, and delayed brain expansion.

\section{Conclusion}

The traumatic SSDH associated with cranial subdural hematoma is a rare case. In case of SSDH, symptomatic treatment will be needed. If there is no neurological deficit, conservative treatment may be a good option. Follow-up evaluation for asymptomatic cranial subdural hematoma will also be necessary.

- The authors have no financial conflicts of interest.

\section{REFERENCES}

1) Bortolotti C, Wang H, Fraser K, Lanzino G. Subacute spinal subdural hematoma after spontaneous resolution of cranial subdural hematoma: causal relationship or coincidence? Case report. J Neurosurg 100(4 Suppl Spine):372-374, 2004

2) Braun P, Kazmi K, Nogués-Meléndez P, Mas-Estellés F, ApariciRobles F. MRI findings in spinal subdural and epidural hematomas. Eur J Radiol 64:119-125, 2007

3) Cho DC, Sung JK. Traumatic subacute spinal subdural hematoma successfully treated with lumbar drainage: case report. J Spinal Disord Tech 22:73-76, 2009

4) Greiner-Perth R, Mohsen Allam Y, Silbermann J, Gahr R. Trau- matic subdural hematoma of the thoraco-lumbar junction of spinal cord. J Spinal Disord Tech 20:239-241, 2007

5) Hung KS, Lui CC, Wang CH, Wang CJ, Howng SL. Traumatic spinal subdural hematoma with spontaneous resolution. Spine (Phila Pa 1976) 27:E534-E538, 2002

6) Ji GY, Oh CH, Chung D, Shin DA. Spinal subdural hematoma following cranial subdural hematoma: a case report with a literature review. J Korean Neurosurg Soc 54:515-517, 2013

7) Juvonen T, Tervonen O, Ukkola V, Klintrup HE. Widespread posttraumatic spinal subdural hematoma--imaging findings with spontaneous resolution: case report. J Trauma 36:262-264, 1994

8) Kim K, Katsuno M, Isu T, Mishina M, Yoshida D, Kobayashi S, et al. Concomitant cranial and lumbar subdural hematomas -case report-. Neurol Med Chir (Tokyo) 50:402-404, 2010

9) Kim MS, Chung CK, Hur JW, Lee JW, Seong SO, Lee HK. Spinal subdural hematoma following craniotomy: case report. Surg Neurol 61:288-292, 2004

10) Morris SF, Poynton AR, O'Donnell T, McCormack D. Lumbosacral subdural hematoma following minor trauma. A case report. J Bone Joint Surg Am 86-A:1768-1771, 2004

11) Nagashima $H$, Tanida $A$, Hayashi I, Tanishima $S$, Nanjo $Y$, Dokai $\mathrm{T}$, et al. Spinal subdural haematoma concurrent with cranial subdural haematoma: Report of two cases and review of literature. $\mathbf{B r}$ J Neurosurg 24:537-541, 2010

12) Payer M, Agosti R. Spontaneous acute spinal subdural hematoma: spontaneous recovery from severe paraparesis--case report and review. Acta Neurochir (Wien) 152:1981-1984, 2010

13) Russell NA, Benoit BG. Spinal subdural hematoma. A review. Surg Neurol 20:133-137, 1983

14) Sari A, Sert B, Dinc H, Kuzeyli K. Subacute spinal subdural hematoma associated with intracranial subdural hematoma. J Neuroradiol 33:67-69, 2006

15) Shimada Y, Sato K, Abe E, Miyakoshi N, Tsutsumi Y. Spinal subdural hematoma. Skeletal Radiol 25:477-480, 1996

16) Shimizu S, Tachibana S, Maezawa H, Fujii K, Kan S. Lumbar spinal subdural hematoma following craniotomy--case report. Neurol Med Chir (Tokyo) 39:299-301, 1999

17) Wajima D, Yokota H, Ida Y, Nakase H. Spinal subdural hematoma associated with traumatic intracranial interhemispheric subdural hematoma. Neurol Med Chir (Tokyo) 52:636-639, 2012 